\title{
Acidentes com animais peçonhentos e venenosos em idosos registrados em municípios do estado de Santa Catarina, Brasil
}

\author{
Vilma Beltrame*, Fernanda Maurer D'Agostini**
}

\section{Resumo}

O presente estudo tem por objetivo avaliar aspectos epidemiológicos dos acidentes com animais peçonhentos e venenosos em idosos registrados em municípios do estado de Santa Catarina, na Região Sul do Brasil, por meio de dados do Sistema de Informação de Agravos de Notificação (Sinan). Foram analisadas, de maneira retrospectiva, informações sobre os acidentes ocorridos no período de 2010 a 2014, e os dados foram obtidos por meio das "Fichas de Acidentes por Animais Peçonhentos", atendidos e/ou ocorridos nas unidades de atendimento. Foram registrados 1.656 casos de acidentes, sendo que 246 ocorreram em idosos, 143 do sexo masculino e 103 do sexo feminino. A maioria dos acidentes foi ocasionada na zona rural e causada por aranhas, seguidas de lagartas, abelhas, serpentes, escorpiões e vespas. Quanto à sazonalidade, o período em que ocorreu o maior número de acidentes foi nos meses de dezembro a março. $\mathrm{O}$ tempo de atendimento médico variou de 1 hora a 24 horas. Os dados encontrados permitem diagnosticar a necessidade e a im- portância de estudos epidemiológicos sobre acidentes causados por animais peçonhentos e venenosos.

Palavras-chave: Envenenamento. Epidemiologia. Peçonhento. Sinan.

\section{Introdução}

O Ministério da Saúde considera acidente por animais peçonhentos todo o

[...] caso ocorrido no paciente com evidências clínicas de envenenamento, específicas para cada tipo de animal, independentemente do animal causador do acidente ter sido identificado ou não (BRASIL, 2005, p. 101).

Segundo o Instituto Butantan (2007), animais peçonhentos são aqueles capazes de inocular substância tóxica (serpentes, aranhas, escorpiões). Já os animais venenosos são aqueles que

* Graduada em Enfermagem e Obstetrícia na Universidade do Sul de Santa Catarina. Mestre em Enfermagem pela Universidade Federal de Santa Catarina. Doutora em Gerontologia Biomédica pela Pontifícia Universidade Católica do Rio Grande do Sul. Professora da Universidade do Oeste de Santa Catarina e da Universidade do Contestado. E-mail: vilma.beltrame@unoesc.edu.br

** Graduada em Ciências Biológicas na Pontifícia Universidade Católica do Rio Grande do Sul. Especialista em Sistemática e Biodiversidade Animal na Pontifícia Universidade Católica do Rio Grande do Sul. Mestre em Biociências (Zoologia) pela Pontifícia Universidade Católica do Rio Grande do Sul. Doutora em Zoologia pela Pontifícia Universidade Católica do Rio Grande do Sul. Professora da Universidade do Oeste de Santa Catarina. Endereço para correspondência: Rua Getúlio Vargas, 2125, Bairro Flor da Serra, Joaçaba, SC. CEP: 89.600-000. E-mail: fernanda.dagostini@unoesc.edu.br

$\rightarrow$ http://dx.doi.org/10.5335/rbceh.v14i3.6769

Recebido em: 01.03.2017. Aceito em: 03.04.2017. 
produzem veneno, mas não possuem um aparelho inoculador, provocando envenenamento passivo por contato, por compressão, como as lagartas do gênero Lonomia, ou por ingestão.

Os acidentes com animais peçonhentos e venenosos constituem um sério problema de saúde pública no Brasil. O número de notificações de acidentes com animais peçonhentos vem aumentando ano a ano (BRASIL, 2005). Em 2003, por exemplo, foram registrados 25.478 acidentes com serpentes, 15.038 com aranhas, mais de 21.000 acidentes com escorpiões, 291 acidentes por Lonomia e 820 por outras lagartas. Segundo a Diretoria de Vigilância Epidemiológica (2016), no estado de Santa Catarina, foram registrados nos últimos dez anos, pelo Centro de Informações Toxicológicas, mais de 20.000 casos de acidentes por esses animais.

Estima-se que no mundo ocorram mais de 500 mil casos de ofidismo e cerca de 130 mil óbitos (26\%) por ano. Grande parte desses óbitos ocorre pela falta do soro específico, pelo retardo na sua administração ou pelo uso incorreto do produto. Dessa forma, tais acidentes preenchem o critério de "neglected tropical disease", uma vez que afetam particularmente pessoas pobres e desprovidas de poder político das áreas rurais de países tropicais, com baixa renda per capita (SOUZA, 2010).

No Brasil, o Sistema de Informações de Agravos de Notificação (Sinan), foi criado em 1990, com o objetivo de registrar e processar os dados sobre agravos de notificação em todo o território nacional, fornecendo informações para análise do perfil da morbidade e contribuindo, dessa forma, para a tomada de decisões nos níveis municipal, estadual e federal. O formulário de entrada dos dados no Sinan é composto de dois documentos distintos. O primeiro é a Ficha Individual de Notificação (FIN), que é preenchida a partir da suspeita clínica da ocorrência de algum agravo notificável atendido num serviço de saúde e encaminhada aos serviços responsáveis pela Vigilância Epidemiológica, no nível municipal. $\mathrm{O}$ segundo documento é a Ficha Individual de Investigação (FII), que, na maioria das vezes, é o roteiro de investigação para cada um dos agravos, sendo utilizado de preferência pelos serviços municipais de vigilância (LAGUARDIA et al., 2004).

No Brasil, existem quatro gêneros de serpentes peçonhentas responsáveis pelos acidentes ofídicos: Bothrops, Crotalus, Lachesis e Micrurus. Acidentes por serpentes não peçonhentas são relativamente frequentes, porém não determinam acidentes graves e, por isso, são considerados de menor importância médica.

Os escorpiões de importância médica ocorrentes no Brasil pertencem à família Buthidae, representados pelo gênero Tityus. Três espécies de escorpião do gênero Tityus têm sido responsabilizadas por acidentes humanos: Tytius serrulatus, Tityus bahiensis, e Tityus stigmurus. Em Santa Catarina, acidentes por escorpiões são comuns, sendo provocados pelo Bothriurus bonariensis, ou escorpião preto, porém não possuem interesse toxicológico devido à baixa toxicidade do veneno (TORRES et al., 2002).

No Brasil, existem três gêneros de aranhas, que podem causar envenenamentos 
no ser humano: Latrodectus (viúva negra), Loxosceles (aranha marrom) e Phoneutria (aranha armadeira). O gênero Loxosceles é encontrado em todo o país, mas sua importância é mais destacada na Região Sul, particularmente no Paraná, onde tem proliferado de maneira significativa na última década. Várias são as espécies descritas, mas as principais causadoras de acidentes são: Loxosceles intermedia, $L$. laeta e L. gaucho (BRASIL, 2005).

Os acidentes causados por formas larvárias de insetos lepidópteros, popularmente conhecidos como taturana, ruga, lagarta-de-fogo, constituem assunto pouco estudado na literatura brasileira, embora sejam comuns e gerem quadros clínicos diversos. As principais famílias de lepidópteros causadoras de acidentes são a Megalopygidae e a Saturniidae (CARDOSO; HADDAD JR., 2005; BRASIL, 2005).

A população idosa constitui o segmento que mais cresce, e essa mudança na pirâmide populacional brasileira tem consequência direta no Sistema de Saúde, uma vez que as doenças próprias da população idosa ganham maior prevalência no conjunto da sociedade. E, muitas vezes, observa-se que a demanda nos serviços de saúde passou a não corresponder à necessidade do idoso (LIMA-COSTA; VERAS, 2003; VERAS, 2007).

No Brasil, são poucos os estudos envolvendo acidentes com animais peçonhentos e venenosos, menos ainda estudos com idosos. Em todo o mundo, a proporção de pessoas com 60 anos ou mais de idade está crescendo mais rapidamente que a de qualquer outra faixa etária, logo, torna-se imperativo conhecer os fenômenos que determinam o estado de saúde/doença dessa população, para que se possa planejar as ações de promoção e proteção à saúde. Sendo assim, este estudo tem por objetivo conhecer o perfil epidemiológico dos acidentes com animais peçonhentos e venenosos em idosos registrados no Sinan, ocorridos em municípios do sul do Brasil, no período de 2010 a 2014, analisando o preenchimento das fichas de notificações.

\section{Materiais e métodos}

A região deste estudo - Associação dos Municípios do Alto Uruguai Catarinense (Amauc), localiza-se no meio Oeste de Santa Catarina, tendo o município de Concórdia como sede. A associação é composta pelos municípios de Alto Bela Vista, Arabutã, Arvoredo, Concórdia, Ipira, Ipumirim, Irani, Itá, Lindóia do Sul, Paial, Peritiba, Piratuba, Presidente Castelo Branco, Seara e Xavantina, com uma população residente de 141.990 habitantes. Os idosos somam 18.577 habitantes, $13 \%$ da população total (INSTITUTO BRASILEIRO DE GEOGRAFIA E ESTATÍSTICA, 2010).

O estudo foi de caráter descritivo e exploratório. Para realizar o levantamento epidemiológico dos acidentes com animais peçonhentos e venenosos, foram obtidos os dados notificados na Secretaria de Saúde do Município de Concórdia, a partir do Sinan, no período de 2007 a 2010. Foram consideradas as seguintes variáveis: tipo de acidente, sexo e idade dos pacientes, região anatômica em que ocorreu a picada, tempo decorrido após 
o acidente, classificação do caso (leve, moderado ou grave) e local onde ocorreu $o$ acidente (ambiente rural ou urbano).

Efetuou-se cálculo de frequência (F\%) para cada item do instrumento de pesquisa, conforme a equação a seguir:

$$
\mathbf{F} \%=\frac{\text { Tipo resposta } \mathrm{x} 100}{\text { Total de resposta do item }}
$$

\section{Resultados}

Durante o período de 2010 a 2014 , foram notificados 1.656 acidentes por animais peçonhentos e venenosos, sendo que $246(14,85 \%)$ ocorreram em idosos. Os acidentes em idosos estão distribuídos conforme Tabela 1.

Tabela 1 - Distribuição segundo tipo de acidente e sexo dos pacientes vítimas de acidentes com animais peçonhentos

\begin{tabular}{l|r|r|r|r|r|r}
\hline \multirow{2}{*}{ Tipo de animal } & \multicolumn{2}{|c|}{ Masculino } & \multicolumn{2}{c|}{ Feminino } & \multicolumn{2}{c}{ Total } \\
\cline { 2 - 7 } & \multicolumn{1}{|c|}{$\mathrm{N}$} & \multicolumn{1}{c|}{$\%$} & $\mathrm{~N}$ & \multicolumn{1}{c}{$\%$} & $\mathrm{~N}$ & \multicolumn{1}{c}{$\%$} \\
\hline Aranha & 107 & 43,49 & 74 & 30,08 & 181 & 73,58 \\
Lagarta & 14 & 5,70 & 13 & 5,28 & 27 & 10,98 \\
Abelha & 7 & 2,85 & 8 & 3,25 & 15 & 6,10 \\
Ignorado & 8 & 3,25 & 3 & 12,19 & 11 & 4,48 \\
Serpente & 5 & 2,03 & 2 & 0,82 & 7 & 2,84 \\
Escorpião & 2 & 0,82 & 2 & 0,82 & 4 & 1,62 \\
Vespa & 0 & 0 & 1 & 0,41 & 1 & 0,40 \\
Total & $\mathbf{1 4 3}$ & $\mathbf{1 0 0}$ & $\mathbf{1 0 3}$ & $\mathbf{1 0 0}$ & $\mathbf{2 4 6}$ & $\mathbf{1 0 0}$ \\
\hline
\end{tabular}

Fonte: elaboração das autoras.

Observou-se que os pacientes tinham entre 60 e 97 anos de idade, sendo a faixa etária com maior incidência entre 60 a 69 anos, com 171 indivíduos $(69,52 \%)$, seguida da faixa de 70 a 79 anos, com
55 indivíduos $(22,35 \%)$, da faixa etária de 80 a 89, com 19 indivíduos $(7,73 \%)$, e houve um indivíduo com 97 anos $(0,40 \%)$ (Tabela 2).

Tabela 2 - Distribuição segundo sexo e faixa etária dos pacientes vítimas de acidentes com animais peçonhentos

\begin{tabular}{l|r|r|r|r|r|r}
\hline \multirow{2}{*}{ Sexo/Faixa etária } & \multicolumn{2}{|c|}{ Masculino } & \multicolumn{2}{c|}{ Feminino } & \multicolumn{2}{c}{ Total } \\
\cline { 2 - 7 } & $\mathrm{N}$ & \multicolumn{1}{c|}{$\%$} & $\mathrm{~N}$ & \multicolumn{1}{c}{$\%$} & $\mathrm{~N}$ & \multicolumn{1}{c}{$\%$} \\
\hline 60 a 69 & 96 & 39,03 & 75 & 30,48 & 171 & 69,52 \\
70 a 79 & 35 & 14,22 & 20 & 8,14 & 55 & 22,35 \\
80 a 89 & 11 & 4,48 & 8 & 3,25 & 19 & 7,73 \\
90 a 97 & 1 & 0,40 & 0 & 0,00 & 1 & 0,40 \\
Total & $\mathbf{1 4 3}$ & $\mathbf{5 8 , 1 3}$ & $\mathbf{1 0 3}$ & $\mathbf{4 1 , 8 7}$ & $\mathbf{2 4 6}$ & $\mathbf{1 0 0 , 0 0}$ \\
\hline
\end{tabular}

Fonte: elaboração das autoras. 
A zona rural apresentou a maior ocorrência dos acidentes, com 59,75\%, seguida da zona urbana, com $39,46 \%$, um caso ocorreu na região periurbana e outro não foi informado.

Em relação à região anatômica (Tabela 3), o pé foi o local de maior incidência, com 61 indivíduos $(24,80 \%)$; seguido de mão, com 60 (24,40\%); dedo do pé, com 26 (10,58\%); braço, com 24 (9,75\%); dedo da mão, com 19 (7,73\%); tronco, com 16 (6,50\%); cabeça, com 12 (4,87\%); coxa, com $11(4,47 \%)$; perna, com 9 (3,65\%); antebraço, com $8(3,25 \%)$ (Tabela 3$)$. $\mathrm{O}$ tempo decorrido entre o acidente e o atendimento médico variou de menos de 1 hora $(\mathrm{n}=57 ; 23,17 \%)$ a mais de 24 horas $(\mathrm{n}=30 ; 12,19 \%)$.

Tabela 3 - Distribuição da frequência dos acidentes de acordo com a região anatômica da picada

\begin{tabular}{l|r|r}
\hline \multirow{2}{*}{ Local da picada } & \multicolumn{2}{|c}{ Número de ocorrências } \\
\cline { 2 - 3 } & $\mathbf{N}$ & \multicolumn{1}{c}{$\%$} \\
\hline Pé & 61 & $24,80 \%$ \\
Mão & 60 & $24,40 \%$ \\
Dedo do pé & 26 & $10,58 \%$ \\
Braço & 24 & $9,75 \%$ \\
Dedo da mão & 19 & $7,73 \%$ \\
Tronco & 16 & $6,50 \%$ \\
Cabeça & 12 & $4,87 \%$ \\
Coxa & 11 & $4,47 \%$ \\
Perna & 09 & $3,65 \%$ \\
Antebraço & 08 & $3,25 \%$ \\
TOTAL & $\mathbf{2 4 6}$ & $\mathbf{1 0 0 , 0 0}$ \\
\hline
\end{tabular}

Fonte: elaboração das autoras.

Quanto à sazonalidade, o período em que ocorreu o maior número de aci- dentes foi nos meses de janeiro, com 43 $(17,47 \%)$, fevereiro, com 30 (12,20\%), março, com 37 (15,09\%), e dezembro, com $32(13 \%)$ (Tabela 4).

Tabela 4 - Distribuição da frequência dos acidentes de acordo com os meses do ano

\begin{tabular}{l|r|r}
\hline \multirow{2}{*}{ Meses do ano } & \multicolumn{2}{|c}{ Número de ocorrências } \\
\cline { 2 - 3 } & \multicolumn{1}{|c|}{$\mathrm{N}$} & \multicolumn{1}{c}{$\%$} \\
\hline Janeiro & 43 & 17,47 \\
Fevereiro & 30 & 12,20 \\
Março & 37 & 15,09 \\
Abril & 19 & 7,72 \\
Maio & 12 & 4,87 \\
Junho & 16 & 6,50 \\
Julho & 6 & 2,43 \\
Agosto & 12 & 4,87 \\
Setembro & 14 & 5,70 \\
Outubro & 16 & 6,50 \\
Novembro & 9 & 3,65 \\
Dezembro & 32 & 13,00 \\
TOTAL & $\mathbf{2 4 6}$ & $\mathbf{1 0 0}$ \\
\hline
\end{tabular}

Fonte: elaboração das autoras.

Em relação à distribuição dos pacientes de acordo com a severidade dos envenenamentos, apenas um caso foi considerado grave, ocorrido com Loxosceles, no qual foi aplicado o respectivo soro. O paciente procurou atendimento somente após 24 horas do horário do acidente. Foram considerados moderados 17 casos ( 9 aranhas, 2 ignorados, 4 lagartas e 2 serpentes), sendo que em 11 foram administrados os soros; 228 foram considerados leves, sendo que em 4 pacientes foi administrado soro antiofídico, por se tratar de acidente botrópico. 


\section{Discussão}

Com base no levantamento e na análise das notificações de investigação de acidentes com animais peçonhentos em municípios do estado de Santa Catarina no período de 2007 a 2015 , a maior ocorrência de acidentes se deu com aranhas, seguidas de lagartas e abelhas. As características epidemiológicas dos acidentes reportados no presente estudo refletem o panorama geral encontrado na Região Sul do Brasil em relação aos agentes causadores de acidentes.

O predomínio de acidentes entre indivíduos do sexo masculino, na faixa etária entre 60 e 69 anos, com ocorrências na zona rural, pode ser devido ao contínuo trabalho agrícola dos homens nessa faixa etária. Nesta pesquisa, observou-se a prevalência dos acidentes na zona rural, corroborando dados encontrados por Theakston, Warrell e Griffiths (2003), que relacionam a ocorrência com animais peçonhentos e a atividade agrícola no mundo inteiro.

$\mathrm{O}$ maior registro de acidentes com Phoneutria e Loxosceles pode indicar que essas aranhas adaptam-se facilmente a novos ambientes (BÜRCHELL, 1969). Phoneutria e Loxosceles spp. são comumente encontrados em sapatos, feixes de varas, entre lixo e materiais de construção, bem como em bananeiras, o que pode explicar, em parte, a maior frequência de picadas em pés e mãos (BÜRCHELL, 1969; BARRAVIEIRA, 1998).

Os acidentes causados por lagartas são comuns em todo o território brasileiro (CARDOSO; HADDAD JR.,
2005), provavelmente o aumento dessas ocorrências se dê devido à fragmentação de habitats. Segundo Moraes (2009), a maioria dos lepidópteros não é prejudicial ao homem, porém alguns possuem cerdas urticantes das quais são liberadas toxinas que podem ocasionar irritação local ou até mesmo hemorragias (GARCIA; DANNI-OLIVEIRA, 2007), assim como espécimes do gênero Lonomia. Segundo Moraes (2009), os trabalhadores rurais são as vítimas mais comuns desse tipo de acidente. Oliveira, Wen e Sifuentes (2009) afirmam que, para cada caso notificado em área urbana ou periurbana, quatro ocorrem na área rural.

O maior índice de registros ocorridos por serpentes do gênero Bothrops se dá, provavelmente, por serem capazes de adaptar-se a diferentes tipos de ambientes, sendo possível encontrá-las nos ecossistemas mais variados (BARRAVIEIRA, 1998). Outros estudos, como o de Moreno, Queiroz-Andrade e Lira-Da-Silva (2005), realizado no Acre, e o de Bochner e Struchiner (2004), no Rio de Janeiro, também registraram maior número de acidentes com o gênero Bothrops. Os estudos realizados por Ribeiro, Jorge e Iversson (1995), em São Paulo, e por Moreno, Queiroz-Andrade e Lira-Da-Silva (2005), no Acre, relataram que a região anatômica mais frequentemente atingida por serpentes foi a do pé, corroborando o resultado encontrado neste estudo.

Ao caracterizar o perfil demográfico dos pacientes, observou-se a predominância de acidentes envolvendo pessoas do sexo masculino, corroborando o rela- 
tado nos estudos de Moreno, Queiroz-Andrade e Lira-Da-Silva (2005). Esse resultado pode estar associado, juntamente com a faixa etária mais acometida (entre 60 e 69 anos), ao fato de que são os homens que mais frequentemente realizam atividades relacionadas à agricultura e à pecuária, o que possibilita $o$ encontro com os animais peçonhentos (OLIVEIRA; LEITE, 2011).

Em relação ao local anatômico da picada, os pés são os mais atingidos, seguidos das mãos, corroborando os dados encontrados por Bredt e Litchteneker (2014). Isso se deve ao fato de muitos trabalhadores irem ao campo com pés e mãos desprotegidos, em áreas de ocorrência natural desses animais (OLIVEIRA; LEITE, 2011), e também está relacionado aos hábitos terrestres e defensivos dessas espécies.

O tempo transcorrido entre $o$ acidente e o atendimento é importante para evitar complicações e possíveis óbitos, ou seja, quanto menor o tempo da assistência prestada maiores as chances de não existirem sequelas (SILVA, 2017). No presente estudo, observou-se um tempo estimado de 1 a 24 horas. Oliveira e Leite (2011) abordam a prevalência de atendimentos realizados em até seis horas após a picada. Os idosos apresentam uma maior fragilidade em termos fisiológicos, se comparados aos jovens, portanto, quando expostos ao veneno, sofrem maiores danos teciduais e sistêmicos (SILVA et al., 2015), por isso é de extrema importância a procura de atendimento médico logo após o contato com o animal.

\section{Considerações finais}

Os acidentes com idosos envolvendo animais peçonhentos e venenosos, notificados no Sinan, apresentam prevalência em pacientes do sexo masculino, com idades entre 60 e 69 anos e em áreas rurais. A epidemiologia dos acidentes corrobora o perfil dos acidentes encontrado no Brasil, apresentando maior frequência em trabalhadores rurais do sexo masculino.

O correto preenchimento da ficha de notificação e o tratamento precoce para as vítimas de acidentes são importantes para a eficiência do tratamento e a administração correta do soro específico com o menor tempo possível entre o acidente e os primeiros procedimentos clínicos. No presente estudo, observou-se a omissão de algumas informações, o que acaba por comprometer os resultados e, muitas vezes, deixa dúvidas sobre a real situação epidemiológica do paciente, uma vez que, a partir desses registros, podem ser elaboradas ações de vigilância, adoção de medidas de esclarecimento da população e desenvolvimento de políticas públicas voltadas para a realidade local, promovendo intervenções necessárias para diminuição de agravos e melhorias na prestação de assistência.

Na ficha de notificação, o campo que se refere a "outros animais" é geralmente preenchido de maneira equivocada, uma vez que esse campo engloba desde registros de corte de faca (arma branca) até acidentes com animais peçonhentos, o que pode levar à ambiguidade 
nos registros. A falta de conhecimento dos profissionais da saúde acerca da importância da identificação dos animais dificulta o rápido diagnóstico e $o$ tratamento do paciente.

Além da importância médica e epidemiológica em relação aos acidentes com animais peçonhentos e venenosos, deve-se levar em consideração questões sociais e econômicas, uma vez que, dentre os idosos, a faixa etária mais acometida por esses acidentes foi aquela na qual os indivíduos ainda estão economicamente ativos.

A inclusão de acidentes com animais venenosos e peçonhentos na lista de doenças negligenciadas pode representar um avanço importante na saúde pública no que diz respeito à prevenção e ao tratamento. A correta identificação do agente causador possibilita o tratamento adequado e diminui os casos de limitação temporária e os desfechos com óbito.

\section{Accidents with venomous and venomous animals in the elderly registered in municipalities of the state of Santa Catarina, Brazil}

\section{Abstract}

The present study aims to evaluate the epidemiological aspects of accidents with venomous and venomous animals in elderly people registered in municipalities in the south of Brazil, SC, using data from the Sinan. We retrospectively analyzed information on the accidents caused in the period from 2010 to 2014 and the data were obtained through the "Accident Fi- les for Poisonous Animals", attended and/ or occurred at the service centers. There were 1,656 cases of accidents, of which 246 occurred in the elderly, 143 males and 103 females. Most of the accidents were caused in the countryside by spiders, followed by caterpillars, bees, snakes, scorpions and wasps. As for seasonality, the period in which the greatest number of accidents occurred was from December to March. Regarding the time of medical care, it ranged from 1 hour to 24 hours. The data found allow us to diagnose the necessity and importance of epidemiological studies on accidents caused by venomous and venomous animals.

Keywords: Epidemiology. Poisoning. Sinan. Venomous.

\section{Referências}

BARRAVIEIRA, B. Venenos: aspectos clínicos e terapêuticos dos acidentes por animais peçonhentos. In: BRASIL. Manual de diagnóstico e tratamento de acidentes por animais peçonhentos. Brasília: Ministério da Saúde/ Fundação Nacional da Saúde, 1998. p. 49-53.

BOCHNER, R.; STRUCHINER, C. J. Aspectos ambientais e sócio-econômicos relacionados à incidência de acidentes ofídicos no Estado do Rio de Janeiro de 1990 a 1996: uma análise exploratória. Cadernos de Saúde Pública, Rio de Janeiro, v. 20, n. 4, p. 976-985, ago. 2004.

BRASIL. Ministério da Saúde. Secretaria de Vigilância em Saúde. Guia de vigilância epidemiológica. Brasília: Ministério da Saúde, 2005.

BREDT, C. S.; LITCHTENEKER; K. Avaliação clínica e epidemiológica dos acidentes com animais peçonhentos atendidos no Hospital Universitário do Oeste do Paraná 2008- 2012. Revista do Médico Residente, Curitiba, v. 16, n. 1, jan./mar. 2014.

RBCEH, Passo Fundo, v. I4, n. 3, p. 265-274, set./dez. 2017 
BÜRCHEL, W. Biology and venoms of the most important South American spiders of the genera Phoneutria, Loxosceles, Lycosa and Latrodectus. American Zoologist, Oxford, v. 9, p. 157-159, 1969.

CARDOSO, A. E. C.; HADDAD JR., V. Acidentes por lepidópteros (larvas e adultos de mariposas): estudo dos aspectos epidemiológicos, clínicos e terapêuticos. Anais Brasileiros de Dermatologia, Rio de Janeiro, v. 80, n. 6, p. 571-578, 2005.

OLIVEIRA, R. C.; WEN, F. H.; SIFUENTES, D. N. Epidemiologia dos acidentes por animais peçonhentos. In: CARDOSO, J. L. C. et al. (Org.). Animais peçonhentos no Brasil: biologia, clínica e terapêutica dos acidentes. São Paulo: Sarvier, 2009. p. 6-21.

SOUZA, W. Doenças negligenciadas. Rio de Janeiro: Academia Brasileira de Ciências, 2010.

DIRETORIA DE VIGILÂNCIA EPIDEMIOLÓGICA. Acidentes por animais. 2016. Disponível em: <http://www.dive.sc.gov.br/ conteudos/agravos/publicacoes/AcidentesporAnimais3.pdf. Acesso em: 10 ago. 2015.

GARCIA, C. M.; DANNI-OLIVEIRA, I. M. Ocorrência de acidentes provocado por Lonomia obliqua Walker, no Estado do Paraná, no período de 1989 a 2001. Revista da Sociedade Brasileira de Medicina Tropical, Rio de Janeiro, v. 40, n. 2, p. 242-246, abr. 2007.

INSTITUTO BRASILEIRO DE GEOGRAFIA E ESTATÍSTICA. Estatística. População. Primeiros resultados definitivos do Censo 2010: população do Brasil é de 190.755.799 pessoas. 2010. Disponível em <www.ibge. gov.br/home/presidencia/noticias/noticia_visualiza.php?id_noticia=186\&id_pagina=1>. Acesso em: 23 ago. 2015.

INSTITUTO BUTANTAN. Acidentes por animais peçonhentos. São Paulo, 2007. Disponível em: <http://www. butantan.gov.br/ perguntas.htm>. Acesso em: $20 \mathrm{dez} .2016$.
LAGUARDIA, J. et al. Sistema de informação de agravos de notificação em saúde (Sinan): desafios no desenvolvimento de um sistema de informação em saúde. Epidemiologia e Serviços de Saúde, Brasília, v. 13, n. 3, p. 135-147, set. 2004.

LIMA-COSTA, M. F.; VERAS, R. Saúde pública e envelhecimento. Cadernos de Saúde Pública, Rio de Janeiro, v. 19, n. 3, p. 700-701, jun. 2003.

MORAES, R. H. P. Lepidópteros de importância médica. In: CARDOSO, J. L. C. et al. (Org.). Animais peçonhentos no Brasil: biologia, clínica e terapêutica dos acidentes. São Paulo: Sarvier, 2009. p. 227-235.

MORENO, E.; QUEIROZ-ANDRADE, M.; LIRA-DA-SILVA, R. M. Clinical and epidemiological characteristics of snakebites in Rio Branco, Acre. Revista da Sociedade Brasileira de Medicina Tropical, Uberaba, v. 38, n. 1, p. 15-21, fev. 2005.

OLIVEIRA, H. F. A.; LEITE, R. S. Aspectos clínico epidemiológicos de acidentes com serpentes peçonhentas no município de Cuité, Paraíba. Gazeta Médica da Bahia, Salvador, v. 81 , n. 1, p. 14-19, jan./jun. 2011.

RIBEIRO, L. A.; JORGE, M.T.; IVERSSON, L.B. Epidemiologia do acidente por serpentes peçonhentas: estudo de casos atendidos em 1988. Revista de Saúde Pública, v. 29, n. 5, p. 380-388, jan. 1995.

SILVA, P. L. N. Perfil epidemiológico dos acidentes por animais peçonhentos notificados no estado de Minas Gerais durante o período de 2010-2015 Revista Sustinere, Rio de Janeiro, v. 5 n. 2, p. 199-217, jul./ dez. 2017.

SILVA, K. S. M. et al. Acidentes por animais peçonhentos na terceira idade: dados epidemiológicos (2011 - 2014). Anais Congresso Internacional do Envelhecimento Humano, Campina Grande, PB, v. 2, n. 1, p. 1-12, 2015. 
THEAKSTON, R. D. G.; WARRELL, D. A.; GRIFFITHS, E. Report of a WHO workshop on the standardization and control of antivenoms. Toxicon, Oxford, New York, v. 41, p. 541-557, 2003.

TORRES, J. B. et al. Acidente por Tityus serrulatus e suas implicações epidemiológicas no Rio Grande do Sul. Revista de Saúde Pública, São Paulo, v. 36, n. 5, p. 631-633, out. 2002.

VERAS, R. F. Envelhecimento populacional e as informações de saúde do PNAD: demandas e desafios contemporâneos. Cadernos de Saúde Pública, Rio de Janeiro, v. 23, n. 10, p. 2463-2466, 2007. 